\title{
Effects of Two Different Hip-Knee-Ankle-Foot Orthoses on Postural Stability in Subjects with Spinal Cord Injury: A Pilot Study
}

\author{
Mohammad Samadian ${ }^{1,2}$, Monireh Ahmadi Bani ${ }^{3,4}$, Navid Golchin ${ }^{5}$, \\ Mohammad Ali Mardani ${ }^{4}$, John S. Head ${ }^{6}$, Mokhtar Arazpour ${ }^{3,4}$ \\ ${ }^{I}$ Skull Base Research Center, Loghman Hakim Hospital, Tehran, Iran \\ ${ }^{2}$ Department of Neurosurgery, Loghman Hakim Hospital, Shahid Beheshti University of Medical Sciences, Tehran, Iran \\ ${ }^{3}$ Pediatric Neurorehabilitation Research Center, University of Social Welfare and Rehabilitation Sciences, Tehran, Iran \\ ${ }^{4}$ Department of Orthotics and Prosthetics, University of Social Welfare and Rehabilitation Sciences, Tehran, Iran \\ ${ }^{5}$ Department of Neurosurgery, Firuzgar Hospital, Iran University of Medical Sciences, Tehran, Iran \\ ${ }^{6}$ Institute for Health and Social Care Research, Faculty of Health and Social Care, University of Salford, Salford, UK
}

\begin{abstract}
Study Design: Pilot study.
Purpose: Evaluation of two different hip-knee-ankle-foot orthoses (HKAFOs; medial linkage reciprocating gait orthosis [MLRGO] and isocentric reciprocating gait orthosis [IRGO]) using gait and postural stability analysis in four subjects with spinal cord injury (SCI).

Overview of Literature: To the best of our knowledge, no study has evaluated postural stability in subjects with SCI when using MLRGO and IRGO.

Methods: The relative efficacy of each orthosis was evaluated with relevant gait parameters, and an assessment of postural stability and sway during usage was made. Each analysis was conducted following an appropriate period of training and acclimatization. The gait parameters employed in the study were walking speed, cadence, and endurance; these were recorded and analyzed using current, validated methods. Postural stability was assessed using a verified force plate measurement system, and a modified Falls Efficacy Scale (mFES) was used for the measurement of postural sway and the perceived fear of falling.

Results: Walking speed, cadence, and endurance increased with the use of both HKAFOs. When the two types of HKAFOs were compared, all the parameters showed a slight (but not significant) increase with the use of MLRGO compared with the use of IRGO. In contrast, there were slight but insignificant improvements in postural sway with the use of IRGO. However, although there were no significant differences between the two sets of mFES scores, there was a slightly reduced fear of falling with the use of MLRGO compared with the use of IRGO in the static standing position.

Conclusions: It is noteworthy that meaningful interpretations of results can only be drawn if a larger sample is employed. This pilot study showed no significant data; however, the results indicate that the use of MLRGO is superior to that of IRGO in terms of potential improvement in the mobility and confidence levels of subjects with SCl.
\end{abstract}

Keywords: Isocentric reciprocating gait orthosis; Medial reciprocating gait linkage orthosis; Stability; Fear of falling

Received Nov 4, 2017; Revised Jun 19, 2018; Accepted Jun 19, 2018

Corresponding author: Mohammad Ali Mardani

Department of Orthotics and Prosthetics, University of Social Welfare and Rehabilitation Sciences, Kodakyar Street, Daneshjo Boulevard, Evin, Tehran 1985713834, Iran

Tel: +98-22180010, Fax: +98-22180049, E-mail: mardanimohammadali@yahoo.com 


\section{Introduction}

The use of an orthosis can increase the postural stability while standing and walking in patients with spinal cord injury (SCI), particularly when the orthosis is used in conjunction with a walking aid, such as a forearm crutch [1]. Three main types of orthoses can be used for orthotic gait rehabilitation; these include hip-knee-ankle-foot orthoses (HKAFOs), reciprocating gait orthoses (RGOs), and medial linkage orthoses (MLOs) [2].

Currently, the standard treatment for patients with SCI involves the use of RGOs [3-5], which can potentially improve gait, upright posture, and mobility. Isocentric RGOs (IRGOs) are considered the most effective types of RGOs for improving gait parameters and lowering energy consumption [3,5]. IRGO comprises two knee-ankle-foot orthoses (KAFOs) that are connected laterally to the main trunk support with a push-pull rod (rocker bar). Movable hip joints are also included, which can be locked into extension when the patient is in the standing position. IRGO also includes a link mounted in the pelvic section that enables hip flexion to promote a reciprocal extension within the contralateral hip. The IRGO hip joints also restrict hip motion in the frontal plane and permit reciprocal hip motion in the sagittal plane (Fig. 1).

Recently, a new type of orthosis, the medial linkage RGO (MLRGO), which has been designed to combine the advantages of RGOs and MLOs, was described by Ahmadi Bani et al. [6] in 2015 (Fig. 1). MLRGO includes two gears that are in contact with each other and are attached to

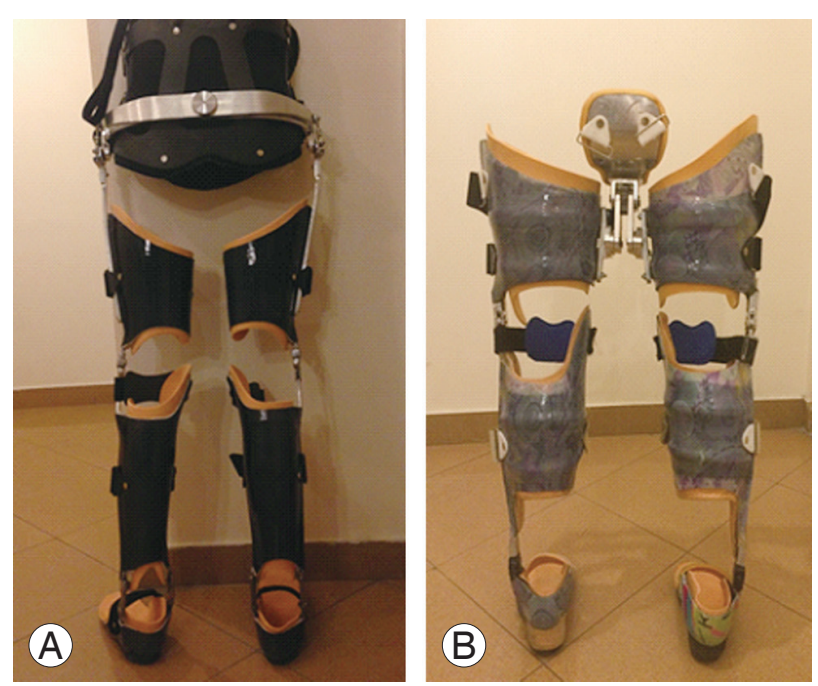

Fig. 1. The orthoses-isocentric reciprocating gait orthosis $(\mathbf{A})$ and medial linkage reciprocating gait orthosis (B) —used in this study. each lower limb. In addition, a saddle is included within the design that is sensitive to pelvic motion and enables the user to activate hip flexion. The axis of rotation is also congruent with the natural hip joint. Furthermore, the more flexible design of this orthosis allows subjects who require lumbar support to combine the use of soft lumbosacral orthoses with MLRGO.

Compared with IRGO, standard MLOs are easier to don/doff and can enable the user to stand up or sit down with relative ease, allowing them to perform activities of daily living more independently. In addition, the more flexible MLO design has greater compatibility with a wheelchair and is visually more appealing than IRGO [7].

MLRGO is designed to include all the advantages of MLO while providing more support and stability than IRGO. MLRGO provides an additional link between each separate KAFO for greater stability [8]; moreover, it is sensitive to pelvic motion and activation and moves the lower extremities reciprocally via the alternate motion of posterior pelvic tilt. Ahmadi Bani et al. [6] in 2015 utilized a 'Lower Limb Paralysis Simulator' to demonstrate that MLRGO could be successfully used as an alternative treatment option to standard RGOs. They also compared the use of IRGO and MLRGO to report that tempo-spatial parameters improved with the use of MLRGO [9].

It is noteworthy that MLRGO is considerably lighter than IRGO (approximately $3 \mathrm{~kg}$ versus approximately 6 $\mathrm{kg}$ ); thus, the MLRGO users can independently don this device more easily, without relying on a helper's assistance. The increased rigidity of IRGO can improve posture; however, this inherent rigidity also limits the specific range of motion in certain joints while standing and during ambulation. In contrast, a semi rigid trunk support is used in MLRGO, which provides more pelvic movement and lower weight. MLRGO also provides lesser physical constraint for users with sensory and motor deficits than that provided by IRGO, with the latter locking the hip joints and inhibiting any type of natural stabilization. In contrast, MLRGO does not include this feature, enabling a more flexible response that helps enhance stability in conjunction with user control.

Postural stability and balance are pre-requisites for all functional activities performed while in the upright stance and are particularly crucial in patients with SCI. However, improving walking parameters (e.g., walking speed and endurance) is the key goal for orthotic rehabilitation. Therefore, combining these characteristics and assessing 
both areas are important for a meaningful analysis of the different types of orthoses and their usability. Thus, this pilot study aimed to investigate the efficacy of IRGO and MLRGO on postural sway, modified Falls Efficacy Scale (mFES), and gait during ambulation in four subjects with SCI, with the objective of undertaking a large-scale study in the future.

\section{Materials and Methods}

Four subjects with SCI and sensory incomplete lesions (American Spinal Injury Association Impairment Scale grade B) were enrolled. Table 1 shows their demographic characteristics. The inclusion criteria were as follows: (1) IRGO use for $>6$ months and the ability to stand independently in the orthosis for at least 90 seconds; (2) successful completion of the gait-training program using MLRGO for 8 weeks (at $1 \mathrm{hr} /$ day for 5 day/wk); and (3) no existing cranial injury, previous lower or upper limb fractures, pressure sores, or psychiatric comorbidities.

Participation was dependent on each subject's complete understanding and agreement of the terms of the study and subsequent signing of the relevant consent form. Ethical approval was granted by the Human Ethical Committee of the Ethical approval was granted by the Human Ethical Committee of the University of Social Welfare and Rehabilitation Sciences.

Each type of orthosis used in this study possessed distinct characteristics and design features. IRGO incorporated drop-lock knee joints and solid ankle joints that were custom-molded for each subject on the basis of the casts of the lower extremity and lumbar region. In contrast, MLRGO (Fig. 1) incorporated two gears that were connected to bilateral KAFOs with a saddle, which were used in conjunction with a soft lumbosacral section. To enable each contralateral swing phase, the saddle-shaped plate of the orthosis is rotated via posterior pelvic tilt. This rotation is transmitted to the gearbox, turning the gear of the medial linkages posteriorly and the swing linkage anteriorly. Thus, an extension rotation of the saddle can generate hip flexion during pelvic swing. By comparison, IRGO was manufactured with the standard hip joint arrangement and single cable for reciprocating gait (Fig. 1).

Each subject received an appropriate level of gait training with MLRGO before the study to compensate for the previous experience of using only IRGO. The training included five 2-hour sessions each week for consecutive 8 weeks. The gait-training program included donning and doffing of the orthosis; passive stretching of the lower extremities; strengthening of the upper limb; standing balance; and walking on regular, flat surfaces. This training was conducted by the same experienced physiotherapist who had treated patients with similar levels of spinal injury. After training, each subject could walk with the new orthosis independently without falling while using a walking aid. The subjects continued to use their prescribed IRGO during the training period.

\section{Outcome measures}

The outcome measures selected for this study were walking speed, cadence, endurance, mean postural sway of the center of pressure (COP) in the medio-lateral (ML) and antero-posterior (AP) directions, and mFES scores.

The relevant gait parameters were assessed with the use of each orthosis during a 6-minute walking exercise performed on even terrain. Each subject was required to walk as naturally as possible using each orthosis in turn. Both walking speed $(\mathrm{m} / \mathrm{sec})$ and cadence (steps $/ \mathrm{min}$ ) were recorded during the second minute of each 6-minute walking session using a hand-held stopwatch. The maximum distance that the subject could walk without resting with-

Table 1. Participant demographics

\begin{tabular}{lccccccc} 
Participant no. & Sex & Age $(\mathrm{yr})$ & Height $(\mathrm{cm})$ & Mass $(\mathrm{kg})$ & Level of injury & AlS grade ${ }^{\text {a) }}$ & Time since injury $(\mathrm{mo})$ \\
Participant 1 & F & 20 & 165 & 54 & T10 & B & 49 \\
Participant 2 & M & 37 & 187 & 85 & T12 & B & 52 \\
Participant 3 & M & 54 & 185 & 95 & T8 & B & 41 \\
Participant 4 & F & 27 & 163 & 58 & T6 & B & 38 \\
Mean & & 34.5 & 175 & - & & & \\
\hline
\end{tabular}

F, female; $M$, male.

${ }^{a)}$ American Spinal Injury Association Impairment Scale grade. 
in the 6-minute period was considered as the endurance measure for each walking activity. A 1-hour rest period was provided between the two orthotic assessments.

A force plate system (Kistler 9286BA; Kistler, Winterthur, Switzerland) was used to measure the effect of postural sway on the position of COP. Subjects were asked to stand at a position that was approximately at the center of the force plate while wearing either IRGO or MLRGO in a random order. Postural sway analyses of each orthosis were performed under two conditions (with two forearm crutches and a single crutch on the dominant side). In each case, the subjects were asked to focus straight ahead on a point $3 \mathrm{~m}$ anteriorly for 30 seconds and to remain silent and focused throughout. This part of the assessment was performed on a distinct day to alleviate the effects of fatigue. The mean amplitude of sway ( $\mathrm{mm}$ ), defined as the mean distance between the sampling points and arithmetic mean point, was calculated where $x i$ and $y i$ represented the difference between COP and the arithmetic mean point at any instant in the AP and ML directions, respectively.

mFES was utilized in this study and was completed following each of the test conditions [10]. mFES uses a Visual Analog Scale to grade 14 different activities, wherein each activity is rated from 1 (lack of confidence and high fear of falling) to 10 (high confidence and low fear of falling). The activities included getting dressed and undressed, preparing a simple meal, taking a bath or a shower, getting in/out of a chair, getting in/out of bed, answering the door or telephone, walking around indoors, reaching into cabinets or a closet, performing light housekeeping, performing simple shopping, using public transport, crossing the road, performing light gardening or hanging out the washing, and using the front or rear steps at home.

\section{Statistical analyses}

A Wilcoxon signed-rank test (non-parametric) was used to analyze the selected outcome measures recorded during the assessment for each orthosis. The IBM SPSS ver. 21.0 program for Windows (IBM Corp., Armonk, NY, USA) was used for this analysis, with a standard significance level of $p \leq 0.05$.

\section{Results}

Table 2 illustrates the mean data sets for the postural sway, relevant gait parameters, and $\mathrm{mFES}$ scores of each subject while walking with each type of orthosis. There was no significant difference in the mFES scores $(p=0.066)$ with the use of the two types of orthoses (Table 3). Walking speed, cadence, and endurance increased with the use of both HKAFOs; the increase was slightly higher with the use of MLRGO than with the use of IRGO. However, these differences were considered insignificant (Table 2). Similarly, there were slight but insignificant differences between the two types of orthoses in terms of postural sway in both the planes during static dual support (ML,

Table 2. Mean postural sway measurements, gait parameters, and mFES scores

\begin{tabular}{|c|c|c|c|c|c|c|c|c|c|}
\hline \multirow{3}{*}{ Participant } & & \multicolumn{4}{|c|}{ Postural sway measurements } & \multirow{2}{*}{\multicolumn{3}{|c|}{ Gait parameters }} & \multirow{3}{*}{$\begin{array}{l}\text { mFES } \\
\text { score }\end{array}$} \\
\hline & & \multicolumn{2}{|c|}{ Dual support } & \multicolumn{2}{|c|}{ Single support } & & & & \\
\hline & & $\begin{array}{l}\text { ML direction } \\
(\mathrm{mm})\end{array}$ & $\begin{array}{l}\text { AP direction } \\
(\mathrm{mm})\end{array}$ & $\begin{array}{l}\text { ML direction } \\
(\mathrm{mm})\end{array}$ & $\begin{array}{l}\text { AP direction } \\
(\mathrm{mm})\end{array}$ & $\begin{array}{l}\text { Speed } \\
(\mathrm{m} / \mathrm{sec})\end{array}$ & $\begin{array}{c}\text { Cadence } \\
\text { (steps/min) }\end{array}$ & $\begin{array}{l}\text { Endurance } \\
(\mathrm{m})\end{array}$ & \\
\hline \multirow[t]{2}{*}{ Participant 1} & A & 38.00 & 35.00 & 40.00 & 45.00 & 0.33 & 24.00 & 102.00 & 50.00 \\
\hline & B & 42.00 & 46.00 & 45.00 & 48.00 & 0.36 & 26.00 & 108.00 & 54.00 \\
\hline \multirow[t]{2}{*}{ Participant 2} & A & 40.00 & 37.00 & 44.00 & 46.00 & 0.31 & 25.00 & 106.00 & 51.00 \\
\hline & B & 44.00 & 44.00 & 48.00 & 46.00 & 0.37 & 28.00 & 114.00 & 55.00 \\
\hline \multirow[t]{2}{*}{ Participant 3} & A & 36.00 & 32.00 & 42.00 & 41.00 & 0.35 & 22.00 & 107.00 & 55.00 \\
\hline & $\mathrm{B}$ & 38.00 & 40.00 & 42.00 & 44.00 & 0.38 & 26.00 & 112.00 & 58.00 \\
\hline \multirow[t]{2}{*}{ Participant 4} & A & 42.00 & 33.00 & 46.00 & 40.00 & 0.32 & 20.00 & 100.00 & 52.00 \\
\hline & B & 45.00 & 42.00 & 49.00 & 43.00 & 0.34 & 24.00 & 110.00 & 57.00 \\
\hline
\end{tabular}

mFES, modified Falls Efficacy Scale; ML, medio-lateral; AP, antero-posterior; A, walking with isocentric reciprocating gait orthosis; $B$, walking with new medial linkage orthosis. 
Table 3. Statistical analysis of postural sway measurements, gait parameters, and mFES scores

\begin{tabular}{|c|c|c|c|c|c|c|c|c|}
\hline \multirow{3}{*}{ Variable } & \multicolumn{4}{|c|}{ Postural sway measurements } & \multirow{2}{*}{\multicolumn{3}{|c|}{ Gait parameters }} & \multirow{3}{*}{$\begin{array}{l}\text { mFES } \\
\text { score }\end{array}$} \\
\hline & \multicolumn{2}{|c|}{ Dual hand support } & \multicolumn{2}{|c|}{ Single hand support } & & & & \\
\hline & $\begin{array}{l}\text { ML direction } \\
\text { (mm) }\end{array}$ & $\begin{array}{l}\text { AP direction } \\
(\mathrm{mm})\end{array}$ & $\begin{array}{l}\text { ML direction } \\
\text { (mm) }\end{array}$ & $\begin{array}{l}\text { AP direction } \\
(\mathrm{mm})\end{array}$ & $\begin{array}{l}\text { Speed } \\
(\mathrm{m} / \mathrm{sec})\end{array}$ & $\begin{array}{c}\text { Cadence } \\
\text { (steps/min) }\end{array}$ & $\begin{array}{l}\text { Endurance } \\
\text { (m) }\end{array}$ & \\
\hline Isocentric RGO & $39 \pm 2.58$ & $34.25 \pm 2.21$ & $43 \pm 2.58$ & $43 \pm 2.94$ & $0.32 \pm 0.017$ & $22.75 \pm 2.21$ & $103 \pm 3.30$ & $52 \pm 2.16$ \\
\hline Medial linkage RGO & $42.25 \pm 3.09$ & $43 \pm 2.58$ & $46 \pm 3.16$ & $45.25 \pm 2.21$ & $0.36 \pm 0.017$ & $26 \pm 1.63$ & $111 \pm 2.58$ & $56 \pm 1.82$ \\
\hline$p$-value & 0.066 & 0.068 & 0.109 & 0.083 & 0.066 & 0.066 & 0.068 & 0.066 \\
\hline
\end{tabular}

Values are presented as mean \pm standard deviation.

mFES, modified Falls Efficacy Scale; ML, medio-lateral; AP, antero-posterior; RGO, reciprocating gait orthosis.

$p=0.066 ; \mathrm{AP}, p=0.068)$ and during single (dominant) support (ML, $p=0.109$; AP, $p=0.083$ ).

\section{Discussion}

The results of this pilot study show no advantages of using MLRGO over IRGO. However, this could be attributed to the considerable heterogeneity among subjects and the relatively low sample size, which makes it challenging to draw a meaningful conclusion regarding the comparative advantages of these two orthoses. Therefore, the results would only involve simple vote-counting, which could provide inaccurate results. To prevent the chances of this error, we decided to perform statistical analyses for the selected parameters. These statistics confirmed that the use of MLRGO fails to demonstrate clear and significant advantages over the use of IRGO within this study population. However, there were some differences between the use of each type of orthosis that may be worth further exploration in a larger-scale study. It is noteworthy that the practical day-to-day usability of MLRGO could be superior because MLRGO is lighter, easier to don/doff, and generally less restrictive and cumbersome than IRGO. It is important to recognize and assess the relative importance of these characteristics and challenge conventional perceptions while considering the best options for successful rehabilitation.

We calculated mean data for postural sway, performing normal activities of daily living when using an orthosis is dependent on attaining postural stability in standing and walking and reducing the fear of falling. Postural stability in this study was assessed using the measure of postural sway. The use of MLRGO in this limited sample size resulted in slightly increased levels of postural sway in both the ML and AP planes compared with the corresponding use of IRGO. However, an analysis of the relevant mFES scores showed that subjects in the static standing position had a slightly lower fear of falling with the use of MLRGO than with the use of IRGO. The inherent reduction in rigidity afforded by MLRGO may have resulted in the subjects' perception of greater control of the orthosis. However, the small sample size and 4-point difference in the mean mFES scores do not allow any tangible significance to be determined at this stage. However, the inclusion of more subjects may give results that could offer greater clinical value.

The higher ability of IRGO to reduce postural sway than that of MLRGO could be attributable to the increased stability afforded by IRGO, particularly around the pelvis and hip joints. In IRGO, the movement of the pelvis and hip joints is limited in all directions. In contrast, MLRGO, which has no pelvic girdle or hip joint, relies on the iliofemoral ligament and hyperextension of the hip joint for providing stability. This interesting analysis reveals that inherent static stability may not always reflect a greater level of dynamic stability.

However, the sample size in the present study is insufficient to support and compare the efficacy of these two types of orthoses on gait parameters and mean walking speed; MLRGO was recorded as $0.32 \mathrm{~m} / \mathrm{sec}$, whereas IRGO was slightly faster at $0.36 \mathrm{~m} / \mathrm{sec}$. These figures were comparable to those achieved by subjects using KAFO with medial linkage in a study by Suzuki et al. [11] in 2007. The use of newer versions of MLOs has shown increases in the walking speed [9], with one type, a 'Primewalk' orthosis, enabling one participant with a T12 level of SCI to produce a mean walking speed of $0.48 \mathrm{~m} / \mathrm{sec}$ [12]. Abe [13] in 2006 compared the walking speeds attained 
with the use of KAFO, walkabout orthosis (WO), and IRGO, respectively, by two subjects with SCI (T12 and T9 levels of injury) and demonstrated that the mean walking speeds differed significantly between the orthoses $(0.05$, 0.08 , and $0.09 \mathrm{~m} / \mathrm{sec}$, respectively). However, it is noteworthy that most studies on orthotic rehabilitation for patients with SCI involve a small sample size with large intra-subject variability, leading to inconclusive results; these variations can be moderated by employing a larger study population. This is particularly relevant when only one participant is employed. Levels of SCI, participant age, and other factors also influence user capability.

The cadence reported in our study was considerably lower than that reported in other similar studies (22-26 versus 40.8-74.1 steps). However, some studies have reported that the mean cadence may vary according to the types of MLO employed. For example, for WO, it was 70.02 steps/min [7] and 40.8 steps/min [14]; with prime walk, it was 48 steps/min [14], 58.9 steps/min [12], 50.9 steps/min [11] and in the hip-ankle-link orthosis, it was 74.1 steps/min [12] for similar subjects with SCI.

\section{Conclusions}

We believe that the results can be meaningfully interpreted only if a larger sample is used. This pilot study did not show any significant data; however, the present results indicate that the use of MLRGO may result in greater improvements in walking speed and endurance in patients with SCI than the use of IRGO, although the differences are too small to be considered significant at this stage. This is also true with respect to the fear of falling. The use of IRGO limited postural sway compared with the use of MLRGO; however, this may prove to be a positive outcome if the subjects can be shown to have higher levels of independence and confidence. Thus, to identify the most suitable orthosis, several factors require assessment. The achievement of an understanding regarding all the factors and suitable prescription analysis is vital for enhancing the quality of life in subjects with SCI. Therefore, followup with a higher number of subjects is an important next step in the analysis of orthotic treatment and rehabilitation outcomes. One of the most important limitations of this study was the relatively small sample size. The present data are inconclusive; however, they provide enough interest to merit future research.

\section{Conflict of Interest}

No potential conflict of interest relevant to this article was reported.

\section{Acknowledgments}

We thank the University of Social Welfare and Rehabilitation Sciences (grant no. 1299) for financial support for this research.

\section{References}

1. Barbeau H, Ladouceur M, Norman KE, Pepin A, Leroux A. Walking after spinal cord injury: evaluation, treatment, and functional recovery. Arch Phys Med Rehabil 1999;80:225-35.

2. Nene AV, Hermens HJ, Zilvold G. Paraplegic locomotion: a review. Spinal Cord 1996;34:507-24.

3. Harvey LA, Davis GM, Smith MB, Engel S. Energy expenditure during gait using the walkabout and isocentric reciprocal gait orthoses in persons with paraplegia. Arch Phys Med Rehabil 1998;79:945-9.

4. Harvey LA, Smith MB, Davis GM, Engel S. Functional outcomes attained by T9-12 paraplegic patients with the walkabout and the isocentric reciprocal gait orthoses. Arch Phys Med Rehabil 1997;78:706-11.

5. Leung AK, Wong AF, Wong EC, Hutchins SW. The Physiological Cost Index of walking with an isocentric reciprocating gait orthosis among patients with $\mathrm{T}(12)$ - L(1) spinal cord injury. Prosthet Orthot Int 2009;33:61-8.

6. Ahmadi Bani M, Arazpour M, Farahmand F, et al. Design and analysis of a new medial reciprocal linkage using a lower limb paralysis simulator. Spinal Cord 2015;53:380-6.

7. Saitoh E, Suzuki T, Sonoda S, Fujitani J, Tomita Y, Chino N. Clinical experience with a new hip-kneeankle-foot orthotic system using a medial single hip joint for paraplegic standing and walking. Am J Phys Med Rehabil 1996;75:198-203.

8. Middleton JW, Sinclair PJ, Smith RM, Davis GM. Postural control during stance in paraplegia: effects of medially linked versus unlinked knee-ankle-foot orthoses. Arch Phys Med Rehabil 1999;80:1558-65.

9. Bani MA, Arazpour M, Farahmand F, et al. The influ- 
ence of new medial linkage orthosis on walking and independence in spinal cord injury patients: a pilot study. Spinal Cord Ser Cases 2016;2:15033.

10. Lam T, Wolfe DL, Eng JJ, Domingo A. Lower limb rehabilitation following spinal cord injury: version 5.0. Vancouver: Spinal Cord Injury Rehabilitation Evidence; 2010.

11. Suzuki T, Sonoda S, Saitoh E, et al. Prediction of gait outcome with the knee-ankle-foot orthosis with medial hip joint in patients with spinal cord injuries: a study using recursive partitioning analysis. Spinal Cord 2007;45:57-63.

12. Genda E, Oota K, Suzuki Y, Koyama K, Kasahara
T. A new walking orthosis for paraplegics: hip and ankle linkage system. Prosthet Orthot Int 2004;28:6974.

13. Abe K. Comparison of static balance, walking velocity, and energy consumption with knee-ankle-foot orthosis, walkabout orthosis, and reciprocating gait orthosis in thoracic-level paraplegic patients. J Prosthet Orthot 2006;18:87-91.

14. Onogi K, Kondo I, Saitoh E, Kato M, Oyobe T. Comparison of the effects of sliding-type and hinge-type joints of knee-ankle-foot orthoses on temporal gait parameters in patients with paraplegia. Jpn J Compr Rehabil Sci 2010;1:1-6. 